Eval uating i deal conbi nations of necktie and Y-shi rt by sel f-or gani zati on map for the coor di nat $i$ on system

\begin{tabular}{|l|l|}
\hline 著者 & Hoshi no Yoshi nobu \\
\hline $\begin{array}{l}\text { j our nal or } \\
\text { publ i cat i on t i t l e }\end{array}$ & $\begin{array}{l}\text { 2010 I EEE I nt er nat i onal Conf er ence on Fuzzy } \\
\text { Syst ens ( FUZZ) }\end{array}$ \\
\hline page range & $1-5$ \\
\hline year & $2010-07$ \\
\hline URL & ht t p: //hdl . handl e. net /10173/656 \\
\hline
\end{tabular}




\title{
Evaluating ideal combinations of Necktie and Y-shirt by Self-organization Map for the coordination system
}

\author{
Yukinobu HOSHINO, Kochi University of Technology
}

\begin{abstract}
We investigated the impression of externals that $\mathrm{Y}$ shirt and the necktie gave the person. The data set to compose the self-organizing map of the result has been extracted. In this paper, we tested the proposing the coordination system that considers relativity about the image data and impressions to the coordination contrast.
\end{abstract}

\section{INTRODUCTION}

$\mathrm{T}$ $\mathrm{HE}$ human impression is influenced by a color and form. Especially, the dress has a big impression, and influence to human images. The KANSEI, which like human emotional sensitivity, has been researched on a lots of companies to designed and propose some ideas for the marketable products. Recently, shopping on internet is easy, which many people buy something from internet. The development of internet has changed to easy process about a question survey. Today's businesses style is growing. In this research, we have survey and analyzed the human impression about a combination of a necktie and Y-shirt. We would like to propose the coordination system, which have respected the human KANSEI images. We have evaluated the human KANSEI of images about necktie and Y-shirt by SD method.

By the experiment, we have taken 'innovative - non innovative' and 'flashy - somberness' binate image words as the first factor. And as the second factor, we have taken 'elegance - indelicacy' and 'beautiful - not beautiful' binate image words. After that, we have regrouped those words to two evaluate axis, the innovative axis and the elegance axis. The final purpose of our research is a development of the coordination system. And to do that, we have to build the positioning map to discuss the coordination process. In this paper, we propose the using SOM (Self-organization Map). To make those maps, we collected data by the pair comparison method from using the image words of factors. SOM need a real variable data, which is during $[-1.0,1.0]$, therefore we used a ranking analyze of Thurston method with 'innovative' 'flashy' 'elegance' and 'beautiful'. As noted before, data of the ranking analyze is closing $[-1.0,1.0]$, and describing a human ranking image, is so easily to make SOM. And it is easy to rebuild SOM by the readjusted ranking data. Those, a user might be select/explain

Yukinobu HOSHINO : Associate Professor,

School of Systems Engineering, Kochi University of Technology

Tosayamada-cho, Kochi, 782-8502, Japan,

Tel: +81-887-53-2213 Fax: +81-887-57-2120,

E-Mail:hoshino.yukinobu $($ kochi-tech.ac.jp,

WWW :http:/www.ele.kochi-tech.ac.jp/hoshino/
$[-1.0,1.0]$ the coordination image by four values, which used the ranking analyze, and rebuild SOM and can check some candidate coordination. By that repeats operation, the candidate coordination would come near to the best combination of necktie and Y-shirt

After that operation, we have to evaluate relativity between user's images and the candidate map, and compare the human image theorem. Finally, we would report all analyze results.

\section{KANSEI EVALUATION}

About some pictures of necktie and Y-shirt, KANSEI, which like human emotional sensitivity, is evaluated by using SD method and those evaluations is translated to design factors. For this translation process is from the factor analysis about a human image. [6] At the first process, we picked up design factors. Design factors are some parts, forms, sizes and colors. A result of a factor analysis is given us score about the factor axis so we can plot those target images on a score map. This score map explains a mean of image. We check the map and analyze the relativity item's design factor and means. But this way can not decide that relativity automatically. We would like to propose the automatically analyze process as the coordination system.

SD method is called semantic differential method, which is the amount of changing on the mean's space. About SD method, analyzers have to collect some adjective words, which are called KANSEI word, about a target object. Those adjective words are opposite adjective words, which are 'bright - dark' and 'elegance - indelicacy' and so on. A difference amount of during binate image words defined 5 or 7 level. Basically, analyzers use the factor analysis, and discover some factorial mean axis and words.

C.E.Osgood has developed SD method as the quantitative measuring method to discover the concept of connotation meaning. This is an appropriate method to take object data by multidimensional analysis about concepts, adjective words and images of target objects. Those target objects are able to explain from several factors, which are constructed by the sensory stimulation and the perceptual concept.

The characteristic of SD-method is embodiment by the adjective about target concept. This is called the controlled association method. Then, it evaluates the validity of the opposite adjective. In that evaluation, meaning measure method is used usually. The process of the measurement is very simple. It uses the sheet of the question, see fig.1. A sheet has been written in opposition adjectives as KANSEI 
words. There is a step-by-step checkbox there. Human subjects checks if it is fitting to the image of the target.

The factor analysis is one kind of the techniques of multivariate analysis. This analysis method is very general in the field of the psychology. Specifically, this method is used for the measurement of the research and the psychological measure to investigate the characteristic of the personality. In a lot of cases, this method is confused with principal component analysis. There is the principal component analysis to build synthetic score from the observational data. However, there is the factor analysis to get an appropriate component from the observational data. In KANSEI, the factor analysis is the most popular analysis method.

\section{EVALUATION EXPERIMENT}

By use of SD method, this questionnaire experiment, which is some evaluation about the combination about necktie and $\mathrm{Y}$-shirt, have 80 human subjects, are 20 s university students.

We prepared 50 picture samples on a questionnaire web site, see fig.1. Before that, a preliminaries experiment is putted on 20 adjust pair by a questionnaire web site. And 16 adjectives were selected as a functional adjective, see table.1. A scale has been 5 levels $(-2,-1,0,+1,+2)$. Human subjects were taking fully answers and enough rest time to evaluate all pictures.

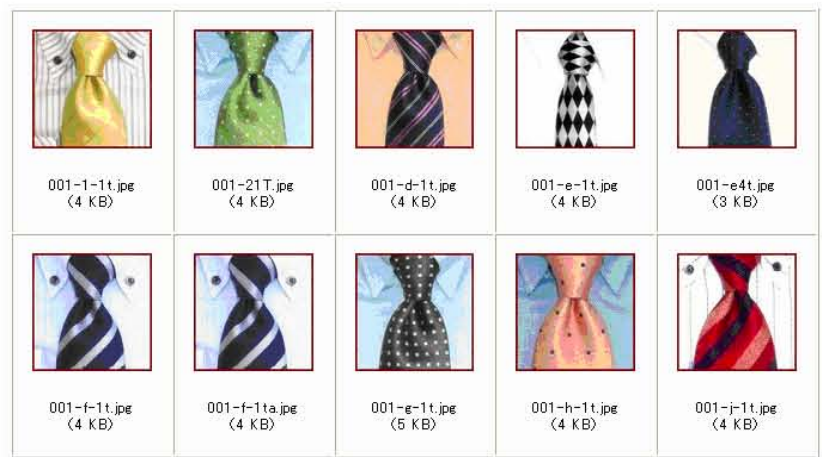

Fig. 1 Image lists for the question form to evaluate

About those data, we applied factor analysis and took factor loadings, given table.2. Number of factor is 2 and analysis uses the varimax rotation.

By the great factor loadings, 'innovative - non innovative' and 'flashy — somberness' are selected for $1^{\text {st }}$ factor axis, and 'elegance — indelicacy' and 'beautiful — not beautiful' are selected for $2^{\text {nd }}$ factor axis. Thus, the name of 1 st axis is defined as the axis of innovative. The name of $2^{\text {nd }}$ axis is defined as the axis of elegance.

From the analysis results, we adopted the set of 'elegance' and 'beautiful' and the set of 'innovative' and 'flashy', which have high common characteristic, as the key word of one pair comparison experiment. Based on the factor score of each item, we made the factor map by plotting each items, is given to fig. 2 .
Table. 1 Check sheet of KANSEI adjective words (Checking example)

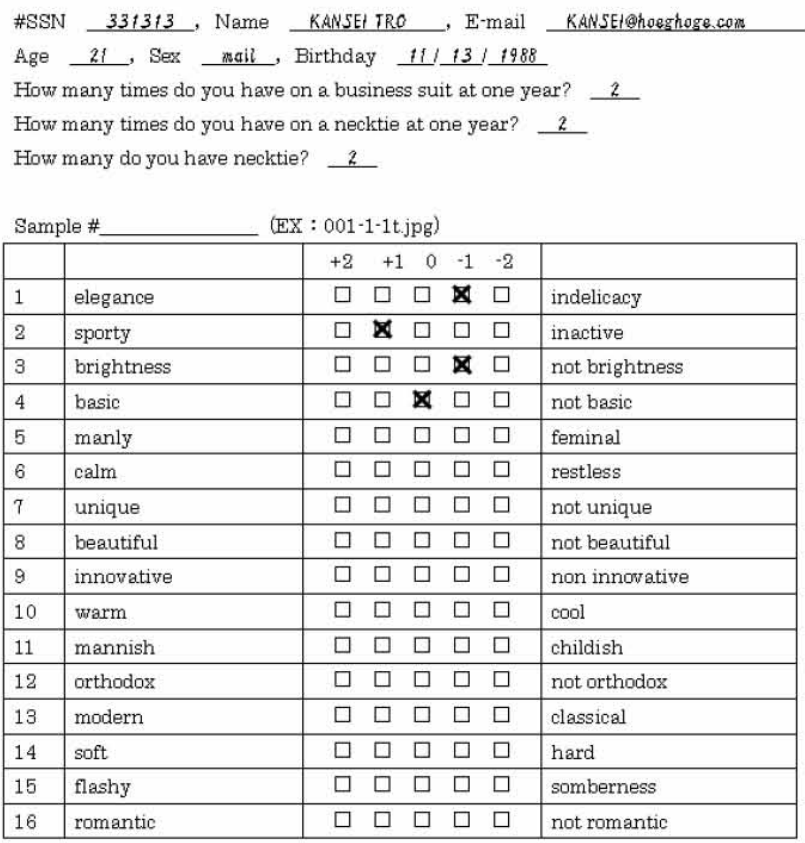

Table. 2 Factor loadings

\begin{tabular}{|c|c|c|c|c|c|}
\hline \multicolumn{2}{|r|}{ Variable } & 1 st factor & 2nd factor & commonality & \\
\hline elegance & indelicacy & 0.140 & -0.754 & 0.588 & 0 \\
\hline sporty & inactive & -0.064 & -0.513 & 0.268 & \\
\hline brightness & not brightness & -0.745 & -0.383 & 0.702 & \\
\hline basic & not basic & 0.682 & -0.399 & 0.624 & \\
\hline manly & feminal & 0.560 & -0.142 & 0.334 & \\
\hline calm & restless & -0.427 & -0.183 & 0.216 & \\
\hline unique & not unique & -0.878 & 0.116 & 0.784 & \\
\hline beautiful & not beautiful & -0.277 & -0.809 & 0.731 & \\
\hline innovative & non innovative & -0.833 & -0.039 & 0.695 & \\
\hline warm & $\mathrm{cool}$ & -0.492 & 0.192 & 0.279 & \\
\hline mannrish & childish & 0.607 & -0.092 & 0.377 & \\
\hline orthodox & not orthodox & 0.712 & -0.492 & 0.748 & \\
\hline moderm & classical & -0.271 & -0.079 & 0.080 & \\
\hline soft & hand & 0.170 & -0.325 & 0.135 & \\
\hline flashy & somberness & -0.859 & 0.006 & 0.738 & 0 \\
\hline momantic & not romantic & -0.373 & -0.478 & 0.367 & \\
\hline & squear of factor loadings & 6.109 & 2.819 & & \\
\hline & contribution $(\%)$ & 33.936 & 15.662 & & \\
\hline & $\begin{array}{l}\text { accumlate of } \\
\text { contribution(\%) }\end{array}$ & 33.936 & 49.598 & & \\
\hline
\end{tabular}

To show the coordination candidacy of the necktie and the Y-shirt using the self-organization mapping about this research is our purpose. For that purpose, the data construction is very important to map for SOM. Because input data of SOM have to be real numbers closing on $[-1.0$, 1.0] evenly, I think.

Therefore, we used one pair pieces of comparison about these key words, the comparative experiments. The examinee is 80 man and women, are 20 's. The result of the comparative experiment uses Thurston analysis and makes ranking data. Ranking data is the real number which is closed on $[-1.0,1.0]$ evenly. Using these data, we create self-organization 
mapping.

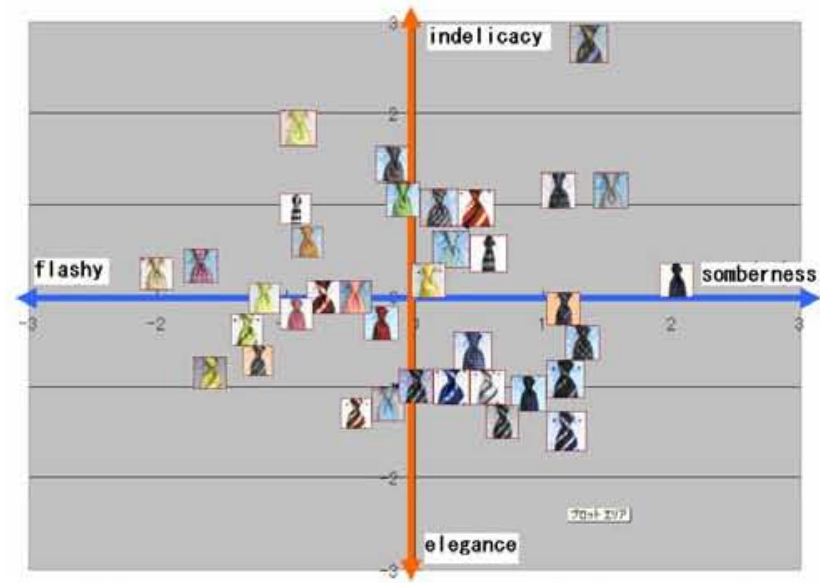

Fig. 2 Score map from the factor analysis

\section{PAIRED COMPARISONTEST}

We picked up the two images which were used by the previous experiment. Then, it prepared those 105 combination images. Therefore, the question of the comparative evaluation is 105 questions, see fig.3. The human subjects are 80 undergraduate students, age is 20 's. The number is as same on the previous experiment. We analyzed this result in ranking by Thurston scaling. Ranking analysis result is shown in fig. 4 and fig. 5 . We could make four indexes using these results for making a self-organization map. It is possible to make a self-organization map using these values.

Quest ion 1

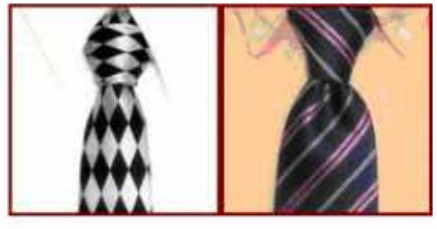

elegance

innovative

beautiful

flashy

$L: 001-e^{-1 t . j p g} \quad R: 001-d-1$ t.jpg

$\begin{array}{ll}\odot & 0 \\ \odot & 0 \\ \odot & 0 \\ \odot & 0\end{array}$

$\odot$

The paired comparison is the way of analyzing a quantitative characteristic about choice of the taste generally. The paired comparison method is used for the research of the psychology and the science. The comparison item is the favorite, the attitude, the voting and the social choice, the public choose and so on. It is used for the field of the psychology literature and $\mathrm{AI}$ of multi-agent. In the psychology, Thurston scale is the beginner technique to measure an attitude. Lewis Leon Thurston developed this scale method in 1928. The problem of the object has some choice statements of paired comparison. Each choice statement has the value which was judged from the favorable alternative, the alternative which is wrong. The human subjects choices an agreement choice on the statement. These choices are totaled and are computed as the score. The average with the score is expressing their attitude. Each average and distribution is computed as scale.

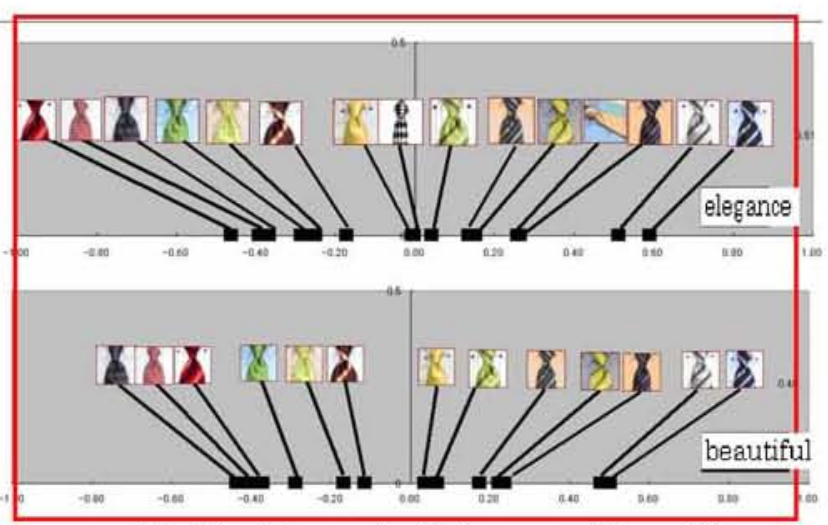

Fig.4 Ranking result of elegance and beautiful

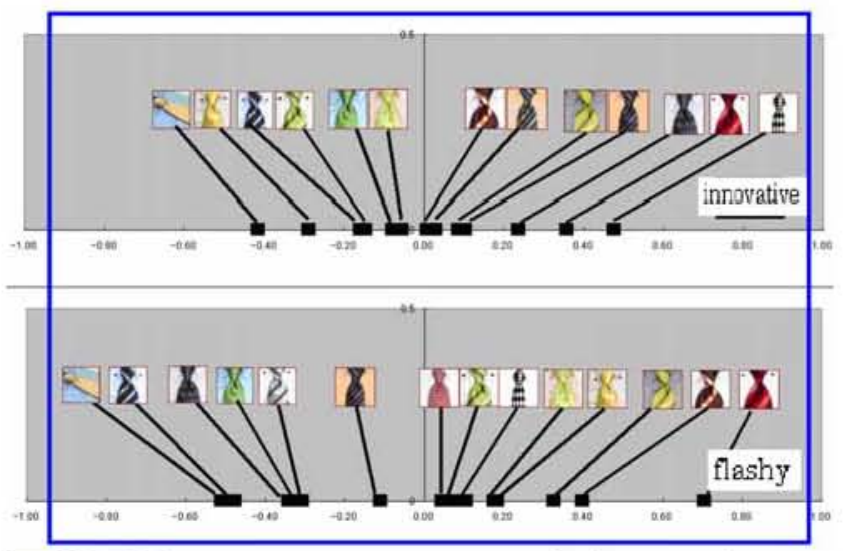

Fig.5 Ranking result about innovative and flashy

\section{SELF-ORGANIZING MAPS}

Self-organizing maps (is called SOM) are a data visualization technique invented by Professor Teuvo Kohonen which reduce the dimensions of data through the use of self-organizing neural networks. [7] Thus multi-dimensional data is able to map two dimensions. The problem that data visualization attempts to solve is that humans simply cannot visualize high dimensional data as is so techniques are created to help us understand this high

Fig.3 Question web site of comparative evaluations

\begin{tabular}{lll} 
elegance & $\odot$ & 0 \\
innovative & $\odot$ & 0 \\
beautiful & $\odot$ & $\circ$ \\
flashy & $\odot$ & $\bigcirc$ \\
\hline \multicolumn{2}{c}{ Fig.3 Question web site of comparative evaluations }
\end{tabular}


dimensional data. Two other techniques of reducing the dimensions of data that has been presented in this course has been multi-dimensional Scaling. SOM go about reducing dimensions is by producing a map of usually two dimensions which plot the similarities of the data by grouping similar data items together. So SOM accomplish two things, they reduce dimensions and display similarities.

SOM is a kind of competitive learning of the no-supervisor type and it is possible to leam by the competition of neighborhood units. After the learning, the weight distribution follows the distribution of the entry data. Therefore, the approximate feature mapping can be made in the data distribution. The mapping which expressed a weight characteristic is displayed as gray scaling data on the two dimensions plane. [1][2] The data which has the characteristic to be the same is mapped in the place which is near respectively. Also, SOM uses gray scaling data, is expressing a correlation value and it becomes mountain and valley structure. SOM can express the position where entry data was mapped, and similarity and a characteristic among the data. Therefore, the person can get visual recognition easily.

\section{THE COORDINATION EXPERIMENT WHICH USED SOM}

SOM is composed of two layers of the input layer and the mapping layer. Generally, all nodes on the mapping layer are arranged in the grid array format. Input layer is made with the entry node of the number of the dimensions which is equal to the entry data number of the dimensions. Weight is given between all the nodes in the mapping layer and all the nodes in the entry layer. This is called connection weight. The entry data is $n$ dimension data. In the early stages of the learning, the influence among the nodes is wide. Weight is learned in the input pattern of the various kinds. By learning progress, the specific win node has an influence on the neighborhood node. In SOM, all the win nodes and all the neighborhood nodes approach an entry vector. In beginning of the learning process, a lot of nodes are made a setup toward with the neighborhood by the neighborhood function and this is formed as a rough map. The weight changes with the progress of the learning process and the number of the neighborhood nodes decreases.

Therefore, slight adjustment with the weight, which is near the local input pattern, is repeated. The weight among the nodes, which are near the characteristic of input pattern, becomes too strong. Then, the weight among the nodes with different characteristic becomes too weak. With these processes, the fellow of the data, which has a characteristic, leads strongly and forms a group. A weight vector is generated to show the characteristic of the whole data. [3][5] The input of SOM is just only those values, which was calculated by Thurston analysis. Therefore, SOM can arrange an item at the map only by the simple data. It is the map for which it is easy to understand the arrangement of the product item for the person. It has analyzed data to the real number which is equal to the evaluation of $[-1.0,1.0]$. Then, to change the image which is expressed by the word is easy. When coordinating, the user can choose the image of the request tie and the request shirt from the real number value $[-1.0,1.0]$ with four items (elegance, innovate, beautiful and flashy) freely. Of course, the user can consult the map, analysis data and ranking result when thinking of the request. We use SOM and can make a new map with ideal data by new adjusted values of the user consideration and all other data of each item. [6] SOM plots the ideal combination of the necktie and the Y-shirt of the sample items and the user image at the new map. The combination image of the necktie and the Y-shirt which was plotted near the ideal combination is coordination candidacy. When the object doesn't like the candidates, user adjusts four items again and creates next other new map again by SOM. This process is repeated until getting the ideal of the user.

\section{Coordination EXPERIMENT 1}

The test subject is one woman of 20 's. First, she watches the factor map on fig. 2 and the ranking analysis results on fig.4-5, and makes ideal image values. She consults the result of ranking analysis and thinks of the combination of the ideal. Each elegance, innovate, beautiful and flashy are chosen from the real number value $[-1.0,1.0]$. We created SOM map from the chose values and each data, shown fig. 6 . The ideal is the very graceful and beautiful combination and the showiness and the novelty might be ordinary. We showed an ideal combination in the plot as new2 to the map of fig.6. An inside area of the closed circle is the ideal combination new 2 and candidacy images in neighboring area of new2, see fig. 6 . The candidacy images approached a comment about the test subject. Therefore, we have taken the ideal candidacy image. As expected, the combination doesn't have innovative images in neighboring area of new2, fig. 6. Controlling values is able to adjustment mapping image and coordination candidacy images. In this experiment, we have some direct questions to adjust value but if the negotiator exists on this coordinate process as a system operator, which is discuss user and decide those values and coordinate by the map.

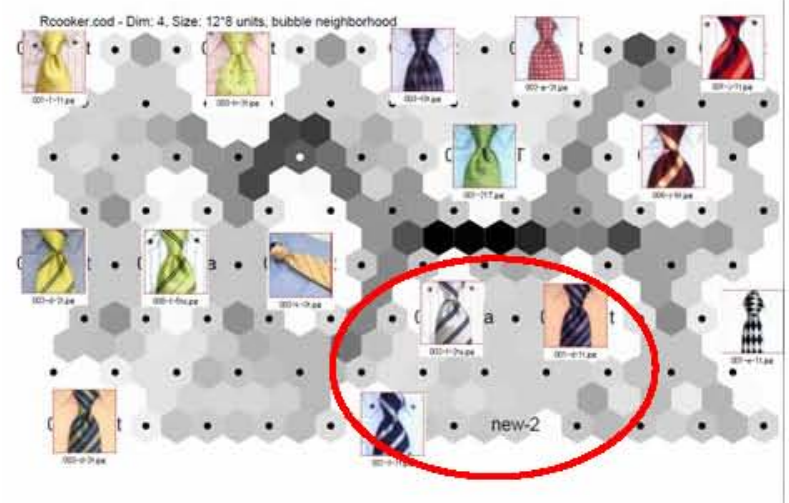

Fig. 6 Coordinate map of experiment 1

\section{Coordination EXPERIMENT 2}

The human subject is one man, age is 20 's. First, he watches the factor map on fig. 2 and the ranking analysis result on fig. $4-5$, and makes ideal image values. He consults the result of ranking analysis and thinks of the combination of the ideal. Each elegance, innovate, beautiful and flashy are 
chosen from the real number value $[-1.0,1.0]$. SOM made the first map from the choicest values and the data of other necktie and Y-shirt. See fig. 7 , which is taken by SOM with his image. The impression of the combination aimed to his image. To adjust values, he checked the around of new 2 on fig. 7 . He said that the combination of my image is more flashy factor only, and no more elegance, innovate and beautiful factors. The part which is surrounded by thick red circle is ideal combination new 2 and the neighborhood of it. $\mathrm{He}$ would like to adjust a little more. By comments about the human subject, we created the second map like fig. 8 once again by adjusted values. On this map, he said that it was permitted for finish his ideal about the neighborhood's images. There are flashy images a little as expected but we can find that combination, which a characteristic isn't arranged.

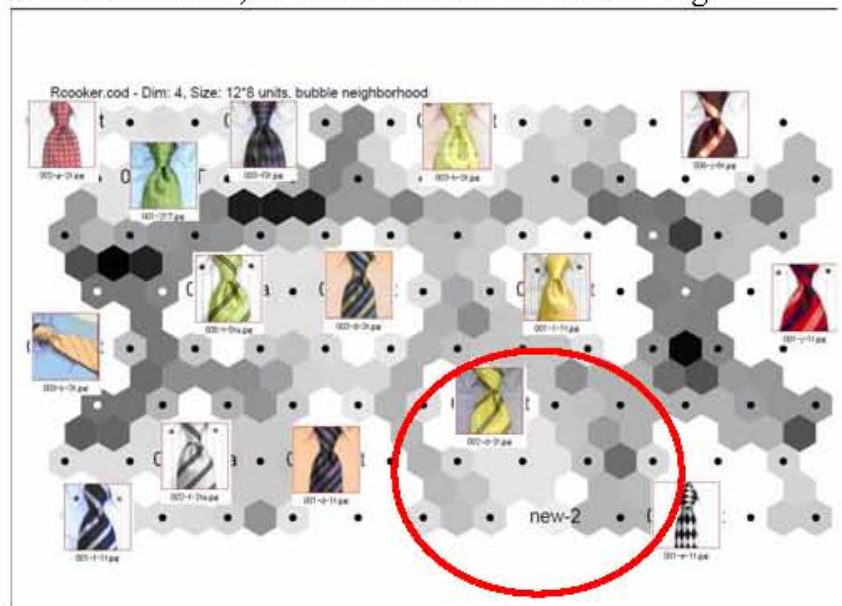

Fig. 7 Coordinate first map of experiment 2

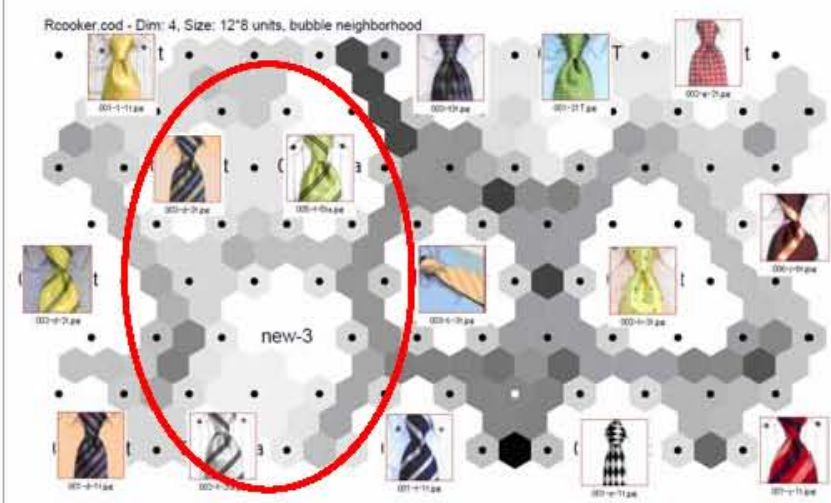

Fig. 8 Coordinate second map of experiment 2

\section{CONCLUSION}

In this paper, we measured the image of the necktie and the $\mathrm{Y}$-shirt in SD-method. Those questionnaire questions used 16 pairs of adjectives. The test subjects are the young students of the man. The images of the young students were shown the score map by the result of analysis of the factor analysis. We discussed about this map and decided the name of the axis. Moreover, we chose the adjective to compose an axis. Using four chosen adjectives, we implemented a questionnaire experiment on one pair pieces of comparison. The result became a meaning scale with Thurston method. As for each value of the adjective, each value enters from the meaning scale to the closed section. This section data has ranking characteristic. We created a product map using the self-organization map. This map shows the users the policy of the combination coordination. We prepared two subjects. The human subject of experiments 1 and 2 is a young man and woman. The examinee can change the value of four adjectives, of innovative, flashy, eleg ance and beautiful about the ideal image. This ideal data and all the image data can be arranged at the same map using SOM. Therefore, we made the nearby image of the ideal data which was gotten in SOM coordination candidacy. The human subject can check the map as the operator and change ideal data to remake the next map. Therefore, we can make a map repeatedly using the changed data. In case of experimentally, the examinee could win the coordination candidacy which is near the ideal, being final. We must consider the usability of the object. Also, our next challenge is to increase the number and the sample of the adjective and to experiment once again. In the future, we plan to think of the improved system design of the operation and then to continue a lot of experiments.

\section{REFERENCES}

[1] Iori Nakaoka Miyuki Matsumura, Jun-ichi Kushida, Katsuari Kamei: "A Proposal of Group Decision Support System for Kansei Commodity Purchase Using SOM and Its Applications", International Journal of Innovative Computing, Information and Control, Vol. 5, No. 12

[2] I. Nakaoka, J.Kushida and K. Kamei: "Proposal of Group Decision Support System Using SOM for Purchase of Automobiles", Proceedings of Third International Conference on Innovative Computing, Information and Control, Dalian, China, pp.C07-07 (2008)

[3] I. Nakaoka, J.Kushida and K. Kamei: "Proposal of Group Decision Support System for Commodity Purchase Using Kansei and SOM", Proceedings of 2008 IEEE International Conference on Systems, Man and Cybernetics, Singapore, pp. 1396-1400 (2008)

[4] I. Nakaoka, M. Matsumura, J. Kushida, K.Kamei: "A Proposal of Group Decision Support System for Kansei Commodity Purchase Using SOM and Its Applications", Proceedings of 2008 International Symposium on Intelligent Informatics, Kumamoto, Japan (2008)

[5] Nakaoka, M. Matsumura, K.Kamei: Negotiation Support System for Decision of Travel Destinations U sing SOM, Proc of The $51^{\text {st }}$ Annual Conference of the Institute of System, Control and information Engineers(Japan) CD-ROM, pp. 623-624 (2007)

[6] S.Nagasawa : "Present State of Kansei Engineering in Japan", Proceedings of 2004 IEEE International Conference on System, Man and Cybemetics, pp. 333 $338,2004$.

[7] T.Kohonen, 「Self-Organizing Map」, Springer (1997) 